\title{
The performance of bootstrapping autoregressive $A R(9)$ process on the Malaysian opening price for second board
}

\begin{abstract}
The commonly used Maximum Likelihood Estimator (MLE) to estimate the parameters of a time series model requires that the process is normally distributed. However, in real situations, many processes are not normal and have a heavy tail distribution. Hence, the aim of this study is to propose using a distribution free bootstrap method for parameter estimations, when the assumption of normality is not met. The performance of the Bootstrap Estimates (BE) and the MLE estimates of the AR (9) process were then investigated using the Malaysian Opening Price for Second Board data and simulation study. The empirical results indicate that the BE is reasonably close to the MLE estimates, hence, can be established as one reliable alternative approach to the MLE estimates.
\end{abstract}

Keyword: AR Process; Bootstrap estimates; Maximum likelihood estimator; Root mean squared errors; Residual bootstrap 